\title{
Intentional and unintentional non- adherence in community dwelling people with type 2 diabetes: the effect of varying numbers of medicines
}

\author{
REBECCA J STACK ${ }^{1}$, CHRISTINE E BUNDY ${ }^{2}$, RACHEL A ELLIOTT ${ }^{3}$, JOHN P NEW $^{4}$, MARTIN GIBSON ${ }^{4}$, \\ PETER R NOYCE ${ }^{5}$
}

\begin{abstract}
Deople with type 2 diabetes are often prescribed multiple medicines which can be difficult to manage. Nonadherence to medicines can be intentional (e.g. active decision) or unintentional (e.g. forgetting). The objective of this study was to measure intentional and unintentional non-adherence to differing numbers of medicines prescribed in type 2 diabetes. A cross sectional survey using the Morisky medication adherence scale (with intentional and unintentional non-adherence subscales) was completed by 480 people prescribed oral antidiabetic drugs (OADs), antihypertensive agents and statins. A within-subject analysis of variance (ANOVA) showed that intentional non-adherence did not vary between OADs, anti-hypertensives and statins. Intentional non-adherence to statins significantly increased when the number of medicines prescribed was included as a between-subjects variable $(p<0.05)$. Another within-subject ANOVA on unintentional non-adherence found a significant difference between OADs, anti-hypertensives and statins; unintentional non-adherence to OADs was significantly higher $(p<0.05)$. When the number of medicines was added as a between-subject variable unintentional non-adherence was associated with higher numbers of medicines. This study shows the difference between intentional and unintentional non-adherence behaviours, and the effect that varying numbers of medicines can have on these behaviours.
\end{abstract}

Br J Diabetes Vasc Dis 2010;X:XXX-XXX.

'School of Social Work, Psychology and Public Health, Salford University, Salford, UK

${ }^{2} S$ chool of Medicine, University of Manchester, Manchester, UK.

${ }^{3}$ School of Pharmacy, University of Nottingham, Nottingham, UK.

${ }^{4}$ Salford Royal Foundation Trust, Salford, UK.

${ }^{5}$ School of Pharmacy, University of Manchester, Manchester, UK.

Correspondence to: Rebecca Stack

School of Social Work, Psychology and Public Health, Salford University,

M6 6PU

Tel: +44 (0)161295 53486<AQ1>

E-mail: r.j.stack@salford.ac.uk
Abbreviations and acronyms
ANOVA analysis of variance
$\mathrm{Cl} \quad$ confidence interval
CVD cardiovascular disease
GCSE General Certificate of Secondary Education
HND Higher National Diploma
OAD oral antidiabetic drug

Key words: adherence, co-morbid, compliance, decisionmaking, polypharmacy

\section{Background}

Co-morbid type 2 diabetes

Seventy percent of adults with type 2 diabetes also have hypertension or hyperlipidaemia. ${ }^{1}$ Subsequently type 2 diabetes is associated with premature CVD² and the risk of CVD mortality is higher for people with type 2 diabetes. ${ }^{3-5}$ Furthermore, the UK's largest cause of mortality is $C V D,{ }^{6}$ and some argue that a large proportion of these cases are attributed to type 2 diabetes. ${ }^{7}$

The impact of polypharmacy in co-morbid diabetes Large randomised controlled trials such as the United Kingdom Prospective Diabetes Study ${ }^{8}$ and the Collaborative Atorvastatin Diabetes Study ${ }^{9}$ advocate intensive pharmacological management in type 2 diabetes. Most people with type 2 diabetes are prescribed multiple medicines to improve metabolic control, lower serum glucose, cholesterol and blood pressure ${ }^{10,11}$ However, many studies have found that adherence is affected by the frequency of doses and the number of medicines prescribed. ${ }^{12}$ For example, Donnan and colleagues found that once daily doses of OADs were associated with better adherence than multiple daily doses..$^{12,13}$ Few studies have examined the effect of multiple medicines from different drug categories. Our previous research has shown that people managing type 2 diabetes and CVD may prioritise some medicines over others, with medicines for hyperlipidaemia management being perceived as less important. ${ }^{14}$ When multiple medicines are prescribed, adherence may vary between medicines as a function of the importance patients give them. 


\section{What is non-adherence?}

Behaviourally, performing health-promoting activities (particularly medicine taking) that follow the advice of a healthcare professional can be defined in multiple ways. Non-adherence and non-compliance are terms that can be applied to behaviours such as not initiating treatment, not taking medicines as prescribed and terminating treatment prematurely. The World Health Organisation has promoted the use of the term 'adherence' in chronic illness, as it denotes a willingness to take medicines. ${ }^{15}$ The term 'adherence' implies that those prescribed medicines are intelligent, active decision-makers, independent and able to take an active role in the management of their condition. ${ }^{16}$ Adherence, like many other health behaviours, is a dynamic and multi-faceted concept.

\section{Types of non-adherence to medicines}

Intentional and unintentional non-adherence are two types of non-adherence behaviours. ${ }^{17}$ Intentional non-adherence is a behaviour driven by a decision not to take medicines. The drivers of this decision are complex but have been suggested to be based on beliefs, personal circumstances, interpretations of healthcare advice and personal motivation. Unintentional nonadherence reflects a person's ability and skill at medicine taking including forgetting, poor manual dexterity, losing medicines or not being able to afford medicines. ${ }^{18}$ Few studies of nonadherence in type 2 diabetes take the number of medicines prescribed into account, or distinguish between intentional and unintentional non-adherence. For example, a cross-sectional study showed $57 \%$ adherence to glucose-lowering medication, $64 \%$ adherence to lipid-lowering medication and $77 \%$ adherence to anti-hypertensive medication in participants. ${ }^{19}$ However, this study did distinguish between intentional or unintentional non-adherence. Intentional non-adherence may have played a larger role in overall non-adherence some medications. More research is needed to investigate the effect of different types of non-adherence across medicines prescribed to those with type 2 diabetes.

\section{Aim}

The aim of this study was to examine intentional and unintentional non-adherence mechanisms across increasing numbers OADs, anti-hypertensives and statins in patients with type 2 diabetes.

\section{Method}

Cross-sectional data were collected from a postal questionnaire and medical records between September 2007 and March 2008. This study was approved by a local National Health Service Research Ethics Committee in May 2007 (reference number 07/Q1410/28).

\section{Participants}

Eight hundred and seventy-eight people with type 2 diabetes and CVD were identified from a secondary care diabetes database. Adult patients (aged 18 years and over) were included if they were prescribed one or more $O A D$, one or more anti-hypertensive, and a statin. People with a care or nursing home address were excluded as they were assumed to have less personal control over taking their medicines than community dwelling populations. People who had not attended the diabetes clinic at the hospital where the study was undertaken within 12 months of the study starting were not approached. This technique improved the response rate and ensured that people were not sent mail from healthcare services with which they had little or no contact.

\section{Materials}

The number of medicines prescribed for type 2 diabetes, hypertension and hyperlipidaemia was extracted from the local hospital's diabetes register. The number of medicines prescribed was categorised as 3, 4 or 5 , and $\geq 6$.

Intentional and unintentional non-adherence across OADs, anti-hypertensives and statins was assessed using the Morisky Medication Adherence Scale (see table 1). A five-point Likert scale was used with scores ranging from 0 (total adherence) to 4 (indicating non-adherence). The Morisky scale measures selfreported intentional (two items) and unintentional (two items) non-adherence to medicines. ${ }^{20}$ This was repeated three times; once for OADs (as shown in table 1), once for anti-hypertensives and once for statins. Therefore, the individual drivers of intentional and unintentional non-adherence for each category of medication could be assessed.

A questionnaire was also used to collect demographic information. The Morisky scale, the demographic information scale and a pre-paid return envelope were posted to potential participants.

\section{Procedure}

The collaborating diabetologist with consent to access patient medical records used the eligibility criteria to identify eligible adults from the diabetes register based on the medicines prescribed. The diabetes register incorporated prescribing information from secondary and primary care to enhance the quality of data obtained. The Morisky medication adherence scale, a participant information

Table 1. The four item Morisky medication adherence scale adapted for type 2 diabetes $^{20 *}$

\section{Morisky questionnaire item}

Item 1 Do you ever forget to take your diabetes medicines?

Item 2 Are you ever careless at times about taking your diabetes medicines?

Item 3 When you feel better do you sometimes stop taking your diabetes medicines?

Item 4 Sometimes, if you feel worse when you take your diabetes medicines, do you stop taking them?

*The Morisky questionnaire was repeated three times; once for oral antidiabetic drugs, once for anti-hypertensive agents and again for statins. 
sheet and an invitation letter were sent to eligible subjects. Data from the questionnaires completed and returned were entered into an SPSS database (version 15). ${ }^{21}$

\section{Analysis}

A repeated measures ANOVA was used to test the hypothesis that intentional and unintentional non-adherence would differ between categories of medicines prescribed for type 2 diabetes and CVD: this was the within-subjects analysis. The 'categorical number of medicines' variable was used as a between-subjects variable. The between-subject effect of the number of medicines prescribed was examined across the repeated measures analyses for intentional and unintentional non-adherence.

\section{Results}

\section{Response rate}

Following the first mailing two reminders were sent to increase the response rate, each two weeks apart from one another. The first mailing directly approached 855 people and 480 questionnaires were returned after three postal mailings (one initial mailing and two reminders). Forty-two people returned a blank questionnaire or declined participation directly. The final response rate for this study was $56 \%(n=480)$.

\section{Demographics}

Participants' mean age was 66.3 years (range: 28-94 years), with $64.6 \%$ male (data were missing in three cases); $91.7 \%$ white, $1.8 \%$ Asian, and $0.4 \%$ from other backgrounds. Data on ethnicity were missing for 25 cases. Table 2 shows the range of medicines prescribed to participants. Nearly one fifth $(21.5 \%)$ of

Table 2. Numbers of participants prescribed each medication

\begin{tabular}{llc}
\hline $\begin{array}{l}\text { Category } \\
\text { of medication }\end{array}$ & $\begin{array}{l}\text { Name of } \\
\text { medication } \\
\text { sub-category }\end{array}$ & $\begin{array}{l}\text { Number (\%) } \\
\text { prescribed }\end{array}$ \\
$\begin{array}{l}\text { Oral antidiabetic } \\
\text { drugs }\end{array}$ & $\begin{array}{l}\text { Metformin } \\
\text { Thiazolidinediones }\end{array}$ & $74(80.33 \%)$ \\
Anti-hypertensives & Angiotensin I & $324(67.50 \%)$ \\
& converting enzyme & \\
& (ACE) inhibitors & \\
& Calcium channel & $211(43.96 \%)$ \\
& blockers & \\
& Angiotensin II & $128(26.67 \%)$ \\
& receptor antagonists & \\
& Diuretics & $126(26.25 \%)$ \\
Alpha-blockers & $49(10.21 \%)$ \\
Sulphonylureas and beta-blockers had not been prescribed to study \\
participants
\end{tabular}

participants reported that their annual household income was less than $f 19,999,7.9 \%$ reported that their annual income was above $f 20,000,42.9 \%$ choose not to disclose their annual household income and $27.7 \%$ reported that they were retired. Over one third (35.4\%) of participants reported that they had no educational qualifications. GCSEs and A levels<AQ2 $>$ were reported to be the highest educational achievement by $15.8 \%$ of participants whilst $8.9 \%$ had achieved a HND qualification or undergraduate degree, $0.6 \%$ had achieved a postgraduate qualification and $39.2 \%$ of people did not report their highest educational achievement.

Rate of total adherence across medication categories Overall, across medicines, levels of intentional (deliberate) non-adherence were lower than unintentional (accidental) non-adherence. The percentage of people who did not report any level of unintentional non-adherence to OADs was $37.1 \%$ $(n=178)$; for anti-hypertensives the percentage of people reporting no level of unintentional non-adherence was $52.9 \%$ $(n=254)$ and no level of unintentional non-adherence to statins was $53.1 \%(n=255)$. The percentage of people who reported no intentional non-adherence was $86.9 \% \quad(n=417)$ for OADs, $80.8 \%(n=388)$ for anti-hypertensives and $81.9 \%$ $(n=393)$ for statins.

\section{Unintentional non-adherence}

The within-subject effect for unintentional non-adherence across medicines was significant $F(2,409)=20.201, p \leq 0.001$, partial eta squared $=0.090)$. Unintentional non-adherence significantly varied between OADs, anti-hypertensives and statins. Therefore, unintentional non-adherence was higher for some medicines than for others. Post-hoc analyses revealed that the difference in unintentional non-adherence between OADs and anti-hypertensives was significant (mean difference $=0.404, p \leq 0.001, \mathrm{Cl}(95 \%)$ 0.288-0.521); unintentional nonadherence to OADs was higher. Unintentional non-adherence was significantly higher for OADs when compared with statins (mean difference $=0.341, \mathrm{p}=0.000, \mathrm{Cl}(95 \%)$ 0.209-0.474) . The difference between unintentional non-adherence to antihypertensives and statins was not significant (mean difference $=-0.063, \mathrm{p}=0.287, \mathrm{Cl}(95 \%)-0.181$ to -0.056$)<\mathrm{AQ} 3\rangle$. For people prescribed multiple medicines for type 2 diabetes, unintentional non-adherence to OADs was found to be significantly higher than for any other prescribed medicines.

Participants were allocated to groups based on the number of medicines prescribed: 232 people were prescribed three medicines; 102 people were prescribed four or five medicines; 146 people were prescribed six or more medicines. These categories formed the between-subjects variable.

When the between-subjects variable was added to the analysis of unintentional non-adherence the number of medicines prescribed was found to have a significant effect $(F(2,410)$ $=3.010, p=0.050$, partial eta squared $=0.014)$. Unintentional non-adherence decreased as the number of medicines prescribed increased (see figure 1) but the interaction was not 
Figure 1. Unintentional non-adherence across medicines for participants prescribed at least three medicines

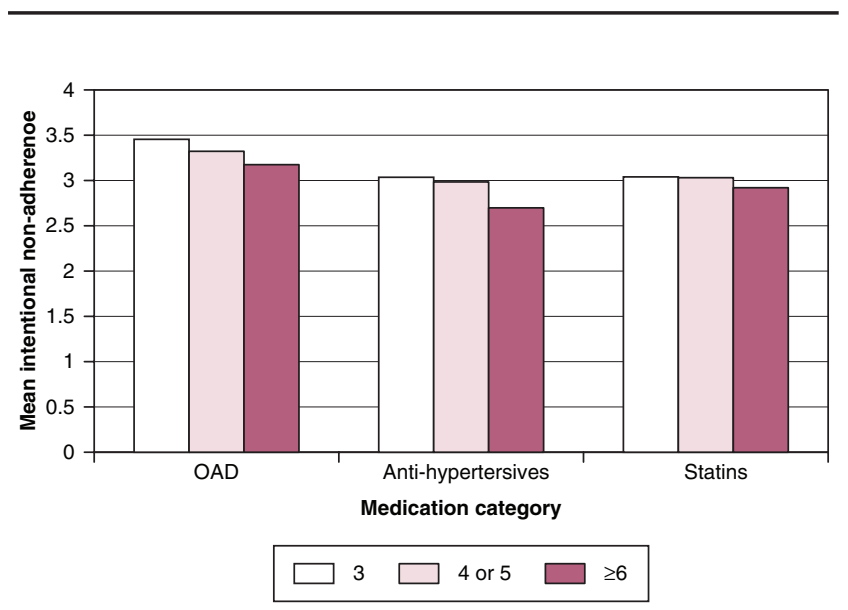

Key: $O A D=$ oral antidiabetic drug

significant $(F(4,818)=0.744, p=0.562)$, therefore, the betweensubjects effect was consistent across all categories of medicines; no one medicine type was particularly affected by the number of medicines prescribed. <AQ3>

\section{Intentional non-adherence}

The repeated measure ANOVA measuring the within-subject effect for intentional non-adherence was not significant $(F(2,410)=2.437, p=0.890)$ therefore intentional non-adherence did not vary between categories of medicines prescribed for type 2 diabetes with CVD. <AQ3>

The between-subjects effect was not significant $(F(2,411)=$ $2.028, p=0.133)$. Therefore, intentional non-adherence did not differ in participants prescribed more medicines. The interaction was significant $(F(4,820)=2.427, p=0.046$, partial eta squared $=0.012$ ) with intentional non-adherence to statins changing as six or more medicines were prescribed. This means that for those taking larger numbers of medicines more decisions not to take statins were made (see figure 2); but this effect did not occur for OADs or anti-hypertensive medicines. $<$ AQ3>

\section{Discussion}

The key finding of this study is that a significant difference in unintentional non-adherence was found between OADs, antihypertensives and statins. Unintentional non-adherence was higher for OADs than anti-hypertensives and statins, while antihypertensives and statins had similar levels of unintentional non-adherence.

This study found that unintentional non-adherence decreased as more medicines were prescribed, suggesting that forgetting decreased for those prescribed greater numbers of medicines. Furthermore, this effect was consistent across all
Figure 2. Intentional non-adherence across medicines for participants prescribed at least medicines

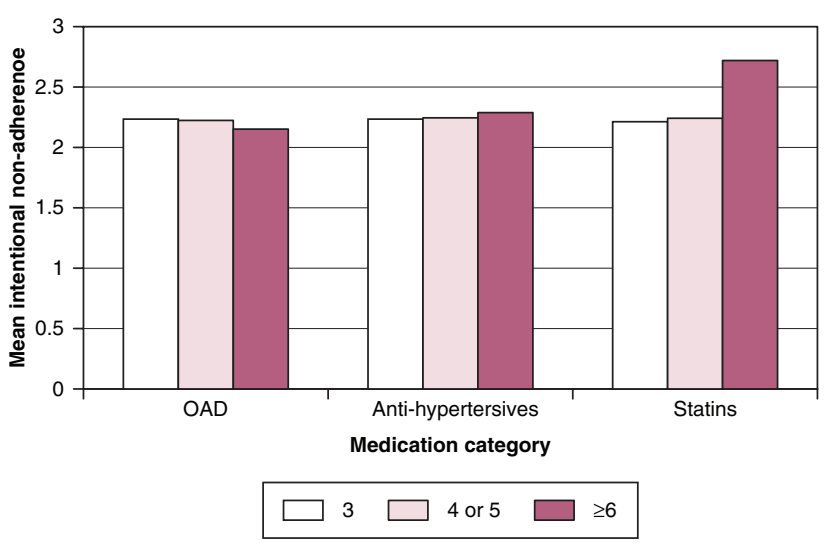

Key: $O A D=$ oral antidiabetic drug

categories of medicines. The possible reason for this finding is that increased vigilance exists among people managing high numbers of medicines. The use of dosette boxes, greater contact with healthcare professionals and greater disease severity may be factors which influence this finding. The range of strategies to manage regimen complexity has been reported elsewhere, ${ }^{22,23}$ it is likely that people used a range of strategies to facilitate adherence in this complex regimen too. Precise examination of these strategies may help improve adherence in complex regimens.

Previous research has drawn the distinction between intentional and unintentional non-adherence. Our research suggests that people with type 2 diabetes and CVD prescribed multiple medicines may vary in their unintentional non-adherence, with medicines for type 2 diabetes being the most likely to be forgotten. Less variance between medicines was observed for intentional non-adherence, however, when the number of medicines prescribed was high greater variance was observed, particularly around taking statins. This study shows that deliberate and accidental non-adherence varied by the number of medicines prescribed, with deliberate non-adherence increasing for statins only, and accidental non-adherence decreasing as more medicines were prescribed.

Unintentional non-adherence was higher for OADs; this may be because medicines for type 2 diabetes are more likely to be prescribed as multiple doses. The increased number of doses for OADs may have increased missed doses; further research should examine whether people have difficulties managing midday doses (possibly because of work or social commitments). Further research should also focus on whether the differences in intentional and unintentional non-adherence are maintained when daily doses as opposed to the number of medicines prescribed are examined. 


\section{Key messages}

- Intentional and unintentional non-adherence are different behaviours

- Unintentional non-adherence differs between medicines

- Intentional non-adherence differs between increasing numbers of medicines

As more medicines were prescribed, non-adherence to statins increased in this study; statins were the only medication to remain consistent across the sample obtained; all participants were prescribed one type of statin. OADs and antihypertensives did increase in number, but remained unaffected by non-adherence decisions. Statins appear to be vulnerable to non-adherence decisions when increasing numbers of medicines are prescribed. Our previous research found that OADs and hypertensive medicines are prioritised above statins by patients. It seems as regimens become more complex then medicines which are undervalued become more vulnerable to non-adherence ${ }^{14}$ Clinicians should recognise that by intensifying the management of type 2 diabetes by increasing OADs or anti-hypertensives, adherence to statins may be compromised.

\section{Limitations}

The participants were from a narrow range of ethnic backgrounds, limiting the generalisability of these findings. The sample was drawn from a population of largely elderly people (mean age 66.3 years) who had a range of long-term conditions. The focus of this investigation was type 2 diabetes and CVD but the people who participated may have been taking other medicines; therefore, the presence of other co-morbidities may have influenced the way medicines were managed for some participants. In addition, there are limitations in the use of self-report measures to examine adherence; over-reporting of non-adherence may have been a problem. Also, some participants may have felt threatened by they way that questions on the Morisky adherence scale are phrased. For example, one question indicates carelessness, which could be interpreted by participants as an accusation or culpability for poor self-management. The potential for questionnaire items to generate negative feeling among participants may have led to underreporting of non-adherence on some questionnaire items. Hypothetically there may be implications of repeating questions on non-adherence for three types of medicines. However, further research is needed to examine the effect of repeated questioning on the reliability and validity of responses to adherence items.

Future research should make attempts to distinguish between intentional and unintentional non-adherence and specify which type of adherence is being measured.

\section{References}

1. Mykkanen L, Kuusisto J, Pyorala K, Laakso M. Cardiovascular disease risk factors as predictors of type 2 (non-insulin-dependent) diabetes mellitus in elderly subjects. Diabetologia 1993;36:553-9.

2. Krentz A, Lawrence J, Summers L. Diabetes and Cardiovascular Disease. Oxford: Churchill Livingstone, 2005.

3. Haffner SM, Lehto S, Ronnemaa T, Pyorala K, Laakso M. Mortality from coronary heart disease in subjects with type 2 diabetes and in nondiabetic subjects with and without prior myocardial infarction. $N$ Engl J Med 1998;339:229-34.

4. Booth GL, Kapral MK, Fung K, Tu JV. Recent trends in cardiovascular complications among men and women with and without diabetes. Diabetes Care 2006;29:32-7.

5. Booth GL, Kapral MK, Fung K, Tu JV. Relation between age and cardiovascular disease in men and women with diabetes compared with non-diabetic people: a population-based retrospective cohort study. Lancet 2006;368:29-36.

6. Beaglehole R, Yach D. Globalisation and the prevention and control of non-communicable disease: the neglected chronic diseases of adults. Lancet 2003;362:903-08.

7. Matthews DR, Pramming S. Diabetes and the global burden of noncommunicable disease. Lancet 2003;362:1763-4.

8. Bretzel RG, Voigt K, Schatz H. The United Kingdom Prospective Diabetes Study (UKPDS) implications for the pharmacotherapy of type 2 diabetes mellitus. Exp Clin Endocrinol Diabetes 1998;106: 369-72.

9. Colhoun HM, Betteridge DJ, Durrington PN et al. Primary prevention of cardiovascular disease with atorvastatin in type 2 diabetes in the Collaborative Atorvastatin Diabetes Study (CARDS): multicentre randomised placebo-controlled trial. Lancet 2004;364:685-96.

10. Morris AD. The reality of type 2 diabetes treatment today. Int J Clin Pract Supp/ 2001;(121):32-5.

11. Rosenstock J. Management of type 2 diabetes mellitus in the elderly: special considerations. Drugs Aging 2001;18:31-44.

12. Paes $A H$, Bakker $A$, Soe-Agnie CJ. Impact of dosage frequency on patient compliance. Diabetes Care 1997;20:1512-17.

13. Donnan PT, MacDonald TM, Morris AD. Adherence to prescribed oral hypoglycaemic medication in a population of patients with Type 2 diabetes: a retrospective cohort study. Diabet Med 2002;19:279-84.

14. Stack RJ, Elliott RA, Noyce PR, Bundy C. A qualitative exploration of multiple medicines beliefs in co-morbid diabetes and cardiovascular disease. Diabet Med 2008;25:1204-1210.

15. World Health Organization. Adherence to long-term therapies. Evidence for action. Geneva: World Health Organization, 2003.

16. Lutfey KE, Wishner WJ. Beyond "compliance" is "adherence" Improving the prospect of diabetes care. Diabetes Care 1999;22:635-9.

17. Clifford S, Barber N, Horne R. Understanding different beliefs held by adherers, unintentional nonadherers, and intentional nonadherers: application of the Necessity-Concerns Framework. J Psychosom Res 2008;64:41-6.

18. Branin JJ. The role of memory strategies in medication adherence among the elderly. Home Health Care Serv Q 2001;20:1-16.

19. Pladevall M, Williams LK, Potts LA et al. Clinical outcomes and adherence to medications measured by claims data in patients with diabetes. Diabetes Care 2004;27:2800-05.

20. Morisky DE, Green LW, Levine DM. Concurrent and predictive validity of a self-reported measure of medication adherence. Med Care 1986;24:67-74.

21. SPSS inc. Statistical Package for the Social Sciences. Chicago: SPSS Inc., 2007.

22. Elliott RA, Ross-Degnan D, Adams AS, Safran DG, Soumerai SB. Strategies for coping in a complex world: adherence among older adults with chronic illness. J Gen Intern Med 2007;22:805-10.

23. Pound $P$, Britten $N$, Morgan $M$ et al. Resisting medicines: a synthesis of qualitative studies of medicine taking. Soc Sci Med 2005;61: 133-55. 